Urologe $2020 \cdot 59: 1560-1564$

https://doi.org/10.1007/s00120-020-01412-5

Online publiziert: 25 . November 2020

(c) Springer Medizin Verlag $\mathrm{GmbH}$, ein Teil von Springer Nature 2020

\title{
G. Knötgen
}

Frauenklinik, Ubbo-Emmius-Klinik Aurich, Aurich, Deutschland

\section{Onkologische Pflege im Tumorboard}

\section{Rolle der Pflege in der multidisziplinären onkologischen Versorgung}

chen Stand entspricht und andererseits die individuellen Voraussetzungen der Patienten berücksichtigt. Dies findet in der Regel einmal wöchentlich interdisziplinär mit verschiedenen Tumorentitäten oder auch als organspezifisch eigenständiges Tumorboard statt. Mögliche Behandlungswege werden auf Grundlage der vorliegenden Informationen diskutiert, ein individueller Behandlungsplan wird erstellt und dokumentiert (Protokoll des Tumorboards).

Um eine flächendeckende, ganzheit che und multidisziplinäre onkologische Versorgung nach validen Qualitätsparametern sicherzustellen, haben die Deutsche Krebshilfe und die Deutsche Krebsgesellschaft (DKG) im Jahr 2008 ein „3-Stufen-Modell der onkologischen Versorgung" definiert. Dieses Zertifizierungssystem zielt darauf $a b$, die Betreuung von Krebspatienten zu verbessern und eine durchgängig an hohen Qualitätsmaßstäben orientierte sektor-, fachdisziplinen- und berufsgruppenübergreifende Behandlung zu garantieren. Dies entspricht den Zielen des Nationalen Krebsplans, Handlungsfeld 2 „Weiterentwicklung der onkologischen Versorgungsstrukturen und der Qualitätssicherung" und Handlungsfeld 4 "Stärkung der Patientenorientierung “ $[9,11,12]$.

Experten aus verschiedenen medizinischen Fachrichtungen erarbeiten dabei individuell eine Therapieempfehlung, die einerseits dem aktuellen wissenschaftli-

Dieser Beitrag wurde erstpubliziert unter Knötgen G (2020) Onkologische Pflege im Tumorboard. Onkologe 26:991-997. https://doi. org/10.1007/s00761-020-00836-0

\section{》) Die fachlichen Anforde- rungen des Erhebungsbogens stellen die Basis für Zertifizierun- gen dar}

Die Durchführung von Tumorboards ist ein zentraler Bestandteil des Zertifizierungsverfahrens Onkologischer Zentren und ist in den Anforderungen des jeweiligen Erhebungsbogens festgelegt. Die fachlichen Anforderungen dieses Erhebungsbogens stellen die Basis für Zertifizierungen von Organzentren, Onkologischen Zentren und Onkologischen Spitzenzentren dar. Diese werden von Zertifizierungskommissionen der DKG entwickelt und jährlich aktualisiert.

Zertifizierungskommission bestehen aus Mandatsträgern der verschiedenen Fachgesellschaften, Arbeitsgemeinschaften, Berufsverbänden und Patientenvertretern. Die KOK als AG der DKG ist ebenfalls pro Kommission mit je einem Mandatsträger beteiligt. Eingereichte Änderungsvorschläge/Überarbeitungen werden in jährlich stattfindenden Sitzungen zur Diskussion gestellt. Nach Konsens der Kommissionsmitglieder wird der Erhebungsbogen entsprechend aktualisiert $[6,8,10]$.

In der Regel werden Tumorboards in Deutschland interdisziplinär mit einem Kernteam aus rein medizinischen Bereichen durchgeführt und entsprechen in diesem Punkt nicht einer ganzheitlichen multidisziplinärem Versorgungsstruktur.

\section{Situation zur Rolle der Pflege im Tumorboard}

Vor November 2018 war eine Teilnahme häufig nur auf Eigeninitiative der Fachpflegenden in der Onkologie und/ oder durch Unterstützung ärztlicher/ pflegerischer Leitungen möglich und reichte von keiner über eine passive bis hin zur aktiven Rolle. Ein Hauptpunkt dafür lag auf dem Passus der Zertifizierungsvorgaben für Punkt 1.8. „Pflege“

- Eine Teilnahme am Tumorboard ist wünschenswert.

und der Vorgabe Punkt 1.2.4 „interdisziplinäre Zusammenarbeit"

- Je nach Bedarf sind weitere Fächer und Berufsgruppen in die Tumorkonferenz einzubeziehen (z. B. Apotheker, Chirurgie, Neurochirurgie, Neurologie, Orthopädie, Palliativmedizin, Pflege, Psychoonkologie, spezielle Schmerztherapie, Nuklearmedizin, Studienkoordination). 


\section{Infobox 1 Aktueller Erhe- bungsbogen für Onkologische Spitzenzentren und Onkologische Zentren (2019) [3]}

Punkt 1.8. Pflege

Patientenbezogene Aufgaben:

- Fachbezogenes Assessment von Symptomen, Nebenwirkungen und Belastungen

- Individuelle Ableitung von Interventionen aus pflegerischen Standards

- Durchführung und Evaluation von pflegerischen und therapeutischen Maßnahmen

- Ermittlung des individuellen patientenbezogenen Beratungsbedarfs

- Im Rahmen des Pflegekonzepts der einzelnen Organzentren ist der fachspezifische Beratungsbedarf bereits zu definieren

- Kontinuierliche Information und Beratung des Patienten (und deren Angehörige) während des gesamten Krankheitsverlaufs

- Durchführung, Koordination und Nachweis von strukturierten Beratungsgesprächen und Anleitung von Patienten und Angehörigen

- Teilnahme am Tumorboard (entsprechend Kap. 1.2)

- Initiierung von und Teilnahme an multiprofessionellen Fallbesprechungen/ Pflegevisiten...

Übergeordnete Tätigkeiten:

- Es ist ein Pflegekonzept zu entwickeln und umzusetzen, in dem die organspezifischen Besonderheiten der onkologischen Pflege in den Organkrebszentren/Modulen Berücksichtigung finden

- Erstellung von fachspezifischen, hausinternen Standards auf Basis von (wenn möglich) evidenzbasierten Leitlinien (z.B. S3-LL Supportiv)

- Angebot einer kollegialen Beratung/ Supervision

- Vernetzung der onkologischen Fachpflegekräfte im OZ (Onkologisches Zentrum), z. B. in gemeinsamen Qualitätszirkeln

- und dort Organisation des Qualifizierungsplans für das $\mathrm{OZ}$ mit seinen Organkrebszentren/Modulen (entsprechend 1.8.5)

- Austausch mit allen an der Behandlung beteiligten Berufsgruppen

„Wünschenswert" und „je nach Bedarf“ bedeuten in der Praxis keine ausreichende Begründung für die Freigabe pflegerischer Ressourcen gerade in Zeiten hoher Arbeitsdichte, dem vorherrschenden Mangel an Pflegepersonal oder gar einer Akzeptanz seitens der Mediziner. Zudem waren bis dato nur eingeschränkt klare Rollen- und Tätigkeitsbeschreibungen für den Bereich Pflege unter Punkt 1.8 festgelegt.

\section{Gründe für eine aktive Rolle der Pflege im Tumorbord}

Dieser Frage hat sich die 2016 gegründete AG Zertifizierung der KOK intensiv gewidmet und hat auf Grundlage der Kompetenzbereiche onkologischer Pflege mehrere Kernpunkte herausgearbeitet. - Pflege ist integraler Bestandteil des multidisziplinären Teams,

- sie bietet ganzheitliche Sicht auf die Patienten,

- pflegerische Kompetenz ergänzt medizinische Kompetenz,

- pflegefachliche Expertise sollte in Empfehlung des Tumorboards einfließen,

- sie fungiert als Patientenvertreter.

Onkologische (Fach-)Pflegende sind die größte Gruppe in der Versorgung der Tumorpatienten in allen Phasen der Erkrankung und sind verstärkt in das Symptommanagement, die Entwicklung der Selbstmanagementkompetenz der Patienten und die Überprüfung der Wirksamkeit der Maßnahmen eingebunden. Ergebnisse aus Studien zu Interventionen von onkologisch Fachpflegenden und deren Wirksamkeit hat die European Oncology Nursing Society (EONS) in Phase 1 des RECaNProjekts (Recognising European Cancer Nursing) als Scoping Review [2] veröffentlicht. Als Schwerpunkte zeichnen sich das Fallmanagement und besonders auch die Beratung und Begleitung im Symptommanagement aus. Eine zur ärztlichen ergänzende pflegerische Aufklärung und Beratung der Patienten über Gesundheits-, Krankheits- und Symptommanagement befähigt die Patienten, fundierte Entscheidungen zu treffen, und kann sie auf die anstehenden komplexen Behandlungen vorbereiten.

Hinsichtlich der aktuellen Pandemiesituation durch COVID-19 sind noch Aufgaben zum Risikomanagement und zur Aufklärung über präventive Maßnahmen wie Social Distancing und Besuchseinschränkungen in Kliniken hinzugekommen. Die jetzt als "systemrelevant“ bezeichnete Berufsgruppe der Pflegen- den mit intensivem Patientenkontakt ist seither konfrontiert mit Ängsten und extremen Verunsicherungen, die zusätzlichen zu der bestehenden Erkrankungssituation auftreten.

\section{》) Durch die Einbringung pflegefachlicher Aspekte werden wertvolle Informationen transparent}

Durch die Einbringung pflegefachlicher Aspekte werden wertvolle Informationen für die weitere Behandlung transparent, die z. B. durchaus auch die Rate an nicht umgesetzten Behandlungsplänen verringern kann. Jalil et al. gingen der Frage nach, wieso Tumorboardempfehlungen im Beobachtungszeitraum nur partiell umsetzt wurden. Am häufigsten als Gründe für die Nichtumsetzung wurden genannt: zu wenig Informationen, (neue) Beurteilung auf Basis von Bildmaterial und pathologischen Befunden, fehlende Berücksichtigung von Patientenwünschen ... sowie Zeitdruck [8].

\section{Änderungen für Pflege im Erhebungsbogen onkologischer Zentren}

Als Änderungsvorlage für die Zertifizierungskommission wurden die pflegerischen Handlungsfelder Punkt 1.8. spezifiziert, mit Nachweispflicht versehen und die Teilnahme am Tumorboard als originäre Tätigkeit definiert (•Infobox 1 ). Fast alle Vorschläge wurden in der Sitzung der Zertifizierungskommission im Konsens positiv bewertet und in den Erhebungsbogen aufgenommen. Allerdings wurde bei der nächsten Aktualisierung im Jahr 2019 zu dem Punkt Teilnahme am Tumorboard der Passus „entsprechend Kap. 1.2", auf Bestreben der Mediziner, hinzugefügt. Die Teilnahme am Tumorbord ist also normativ, jedoch ist Pflege nur ,je nach Bedarf" einzubeziehen.

Zurzeit werden schrittweise onkologisch-pflegerische Kompetenzbereiche (onkologische Pflegeberatung, Pflegekonzept ...) noch genauer definiert, um möglichst als Qualitätskriterium 
Tab. 1 Aufgaben onkologischer Pflege im Tumorboard. (Quelle: G. Knötgen, Rolle der Pflege im Tumorboard, Deutscher Krebskongress 2018)

\begin{tabular}{|c|c|c|}
\hline Vor dem Tumorboard & Während des Tumorboards & Nach dem Tumorboard \\
\hline Erfassung und Bewertung & Pflegefachliche Aspekte & Individuelle pflegefachliche Aufklärung, Beratung \\
\hline $\begin{array}{l}\text { Ganzheitliche Pflegeanamnese (AZ, } \\
\text { Situation, Beschwerden und Ressour- } \\
\text { cen) }\end{array}$ & Zu erwartende Symptome/Begleiterscheinungen & Erläuterung von „fachchinesischen“ Begriffen \\
\hline Assessment von Symptomen & $\begin{array}{l}\text { Einschätzung des Risikopotenzials von Aus-/und } \\
\text { Nebenwirkungen geplanter Therapie im Kontext } \\
\text { mit AZ }\end{array}$ & $\begin{array}{l}\text { Mögliche Auswirkungen geplanter Therapiemaßnah- } \\
\text { men }\end{array}$ \\
\hline $\begin{array}{l}\text { Zu erwartende Aus-/Nebenwirkungen } \\
\text { und Risikofaktoren }\end{array}$ & PatientenorientierteAspekte & Supportive/prophylaktische Maßnahmen \\
\hline $\begin{array}{l}\text { Wünsche, Ängste, Bedürfnisse und } \\
\text { Ziele der Patienten }\end{array}$ & Wünsche und Bedürfnisse & Gezieltes Nebenwirkungsmanagement \\
\hline \multirow[t]{2}{*}{ Erkrankungserfahrungen } & Erwartungen und Ziele & $\begin{array}{l}\text { Bedarfsgerechte Angebote, bzw. Einbeziehung ande- } \\
\text { rer Disziplinen (Sozialdienst, SAPV, Psychoonkologie } \\
\text { etc.) }\end{array}$ \\
\hline & Ethische Aspekte & Förderung von Eigenkompetenz und Adhärenz \\
\hline
\end{tabular}

\begin{tabular}{|c|c|}
\hline Vor der Falldiskuss & \\
\hline $\begin{array}{l}\square \text { Sind die relevante } \\
\text { Tumorboards anwes }\end{array}$ & $\begin{array}{l}\text { Kernmitglieder des } \\
\text { dd? }\end{array}$ \\
\hline $\begin{array}{l}\square \text { Ist jemand anwes } \\
\text { kennt? }\end{array}$ & d, der den Patienten \\
\hline $\begin{array}{l}\square \text { Ist der/die derzeit } \\
\text { Ärztin des Patienten }\end{array}$ & $\begin{array}{l}\text { behandelnde Arzt/ } \\
\text { iwesend? }\end{array}$ \\
\hline Information & Diskussion \\
\hline$\square$ Fallbericht & $\square$ Chirurg \\
\hline$\square$ Komorbiditäten & $\square$ Internist \\
\hline $\begin{array}{l}\square \text { Radiologische } \\
\text { Befunde }\end{array}$ & $\square$ Onkologe \\
\hline $\begin{array}{l}\square \text { Pathologische } \\
\text { Befunde }\end{array}$ & $\square$ Radiologe \\
\hline $\begin{array}{l}\square \text { Psychosoziale } \\
\text { Beurteilung }\end{array}$ & $\square$ Pathologe \\
\hline$\square$ Patientensicht & $\square$ Pflege \\
\hline$\square$ Klinische Studien & $\square$ Palliativteam \\
\hline$\square$ Andere & $\begin{array}{l}\square \text { Medizinische } \\
\text { Assistenzberufe }\end{array}$ \\
\hline Ergebnis & \\
\hline $\begin{array}{l}\square \text { Was sind die Emp } \\
\text { boards? }\end{array}$ & lungen des Tumor- \\
\hline$\square$ Gibt es Bedenken & \\
\hline $\begin{array}{l}\square \text { Muss dieser Patier } \\
\text { tiert werden? }\end{array}$ & och einmal disku- \\
\hline
\end{tabular}

mit Kennzahlen hinterlegt zu werden (- Infobox 1).

Resultierend daraus ergeben sich die pflegerischen Aufgabenbereiche, die eine aktive Rolle im Tumorboard begründen (• Tab. 1).
Die Umsetzung in die Praxis stärkt die Rolle der Pflege in einem multidisziplinären Team und ermöglicht eine Qualitätsverbesserung der onkologischen Versorgung. Sie sorgt ebenfalls für die erforderliche Rollenklarheit zwischen den Teammitgliedern und Rollenidentifikation für den Patienten.

Kommunikation ist dabei genauso essenziell wie die Verfolgung gemeinsamer Ziele in der Behandlung und die Verantwortlichkeit der Teammitglieder für die Erfüllung ihrer jeweiligen Rollen.

\section{Tumorboards im inter- nationalen Vergleich}

Tumorboards (im angloamerikanischen Sprachraum „multidisciplinary team meetings“ genannt, MDT) sind auch international ein anerkanntes obligatorisches Instrument und sind hinsichtlich Multidisziplinarität, Effizienz und Notwendigkeit häufig untersucht worden.

Die European Partnership Action Against Cancer Consensus Group hat hierzu eine Grundsatzerklärung zur multidisziplinären Krebsbehandlung veröffentlicht, in der es heißt, dass der Förderung der Arbeit in multidisziplinären Teams ethische Priorität einzuräumen sei. Borras et al. definieren in dieser Erklärung multidisziplinäre Teams als „Allianz“ von Medizinern und anderen, am onkologischen Behandlungsprozess beteiligten Mitarbeitenden [1, 4].
Der Behandlungsansatz dieser Allianzen ist auf evidenzbasierte Entscheidungen und auf eine koordinierte Patientenversorgung während des gesamten Behandlungsverlaufs ausgerichtet. Dabei werden die Patientinnen und Patienten fortlaufend ermutigt, eine aktive Rolle bei ihrer Behandlung einzunehmen. Therapieentscheidungen sollten unter Heranziehung zusätzlicher Daten getroffen werden. So würden etwa die psychosoziale Situation, die vorhersehbare Lebensqualität, die vorhandenen Begleiterkrankungen oder auch die Erwartungshaltung der Erkrankten berücksichtigt.

Benjamin W. Lamb, et al. ziehen nach ihrer Interventionsstudie folgende Schlussfolgerungen:

Die multidisziplinären Tumorboards (MTB) werden zum weltweiten Standard für die Krebsbehandlung und können mit der Nutzung z.B. der MTBCheckliste (•Tab. 2), des MTB-Teamtrainings und schriftlichen Anleitungen die Qualität der Teamarbeit für die Entscheidungsfindung verbessern und die Krebsbehandlung beschleunigen ([13];

\section{- Tab. 2).}

Die ASCO (American Society of Clinical Oncology) bietet „multidisciplinary cancer management courses“ (MCMC) an und arbeitet dabei mit Organisationen auf der ganzen Welt zusammen, um Schulungen zur Verbesserung der onkologischen Versorgung zu organisieren. MCMC verbessern die Krebsbehandlung weltweit durch Förderung des multidiszi- 
plinären Teammanagements bei Fachärzten, Chirurgen, Pathologen und Mitgliedern des Onkologiepflegeteams [14].

Studien von Fleissig A, Jenkins V, Catt $S$ et al. [5] aus Großbritannien zeigten, dass multidisziplinäre Teams $\mathrm{zu}$ einer besseren Teamdynamik und Kommunikation, einer höheren Patientenzufriedenheit und einem verbesserten klinischen Ergebnis führten.

Die Einführung verschiedener MDTMitglieder wie Physiotherapeuten, Ernährungswissenschaftler, Ergotherapeuten und Sozialarbeiter in das bestehende Team aus Onkologen, Radiologen, Chirurgen, Pflegekräften und Pathologen trug dazu bei, den Informationsaustausch sowie den Diagnose- und Behandlungsprozess zu verbessern $[4,6]$.

\section{》) Multidisziplinäre Teams \\ führten zu besserer Kom- munikation und höherer Patientenzufriedenheit}

Im internationalen Vergleich wird also Pflege, im Gegensatz zu Deutschland, häufig als ein Kernbestandteil des multidisziplinären Tumorboards beschrieben. In der Schweiz wird onkologische Pflege in diesem Kontext teilweise als Cancer Nurse Navigator (CNN) oder im amerikanischen Raum als Oncology Nurse Navigator (ONN) bezeichnet. Im Schweizer Spital Männedorf AG hat die Onkologiepflege sogar die Koleitungsfunktion im Tumorboard.

\section{Fazit für die Praxis}

- Tumorboards sind zentral bei der Planung und Behandlung von Krebserkrankungen und Bestandteil der Zertifizierungsverfahren Onkologischer Zentren.

- Pflegerische Kompetenzen, incl. psychosozialer Unterstützung, Pflegeberatung/Schulung, Pflegekoordination und Patientenbeteiligung, leisten einen wertvollen Beitrag in der onkologischen Versorgungsstruktur.

- Mit multidisziplinärer Zusammenarbeit sind integrierte Diagnosen und Behandlungsempfehlungen zu erreichen, die Daten und Ergebnisse aller beteiligten Spezialisten berücksichtigen.

- Fachpflegende in der Onkologie sind integraler Bestandteil des multidisziplinären Teams und bringen pflegefachliche Aspekte im Kontext mit Patientenwünschen und -bedürfnissen ein, die in die Empfehlung des Tumorboards einfließen können.

- Im internationalen Vergleich wird Pflege als Kernbestandteil des multidisziplinären Tumorboards beschrieben

- Die erfolgten Änderungen in den Zertifizierungsvorgaben sind ein erster Schritt zur Stärkung der Rolle onkologischer Pflege.

\section{Korrespondenzadresse}

G. Knötgen
Frauenklinik, Ubbo-Emmius-
Klinik Aurich
Aurich, Deutschland
knoetgen@
kok-krebsgesellschaft.de

\section{Einhaltung ethischer Richtlinien}

Interessenkonflikt. G. Knötgen gibt an, dass kein Interessenkonflikt besteht.

Für diesen Beitrag wurden von den Autoren keine Studien an Menschen oder Tieren durchgeführt. Für die aufgeführten Studien gelten die jeweils dort angegebenen ethischen Richtlinien.

\section{Literatur}

\section{Verwendete Literatur}

1. Borras JM et al (2014) Policy statement on multidisciplinary cancer care. Eur J Cancer 50:475-480

2. Charalambous A, Wells M, Campbell P, Torrens C, Östlund U, Oldenmenger W, Patiraki E, Sharp L, Nohavova I, Domenech-Climent N, Eicher $\mathrm{M}_{\text {, }}$ Farrell C, Larsson $M$, Olsson C, Simpson $M$, Wiseman T, Kelly D (2018) A scoping review of trials of interventions led or delivered by cancer nurses. Int J Nurs Stud 86:36-43. https://doi.org/10.1016/ j.jinurstu.2018.05.014

3. Deutsche Krebsgesellschaft (2019) Zertifizierung. https://www.krebsgesellschaft.de/deutschekrebsgesellschaft/zertifizierung.html. Zugegriffen:01.08.2020

4. EPAAC (2014) Policy statement on multidisciplinary cancer care https://doi.org/10.1016/j.ejca.2013. 11.012
5. Fleissig A, Jenkins V, Catt Setal (2006) Multidisciplinary teams in cancer care: are they effective in the UK? Lancet Oncol 7:935-943

6. Harris J, Taylor C, Sevdalis N, Jalil R, Green JS (2016) Development and testing of the cancer multidisciplinary team meeting observational tool (MDT-MOT). Int J Qual Health Care 28:332-338

7. Homayounfar K, Lordick F, Ghadimi M (2014) Qualutätssicherung: Multidisziplinäre Tumorboards -trotz Problemen unverzichtbar. Dtsch Arztebl 111(22):A 998-1001

8. Jalil R et al (2013) Factors that can make an impact on decision-making and decision implementation in cancer multidisciplinary teams: an interviey study of the provider perspective. Int J Surg 11:389-394

9. Knötgen G (2019) Onkologische Versorgung multidisziplinär aufstellen. Pflegezeitschrift 72(3):37-39. https://doi.org/10.1007/s41906 019-0019-1

10. KOK Homepage: Kommissionen. https://www. kok-krebsgesellschaft.de/kok/kommissionen/. Zugegriffen:01.08.2020

11. Kowalski C, Wesselmann S (2016) Orientierung in komplexer Versorgungslandschaft. Dtsch Arztebl 113(6):39-40

12. Krebsgesellschaft https://www.krebsgesellschaft. de/zertdokumente.html.Zugegriffen:01.08.2020

13. Lamb BW et al (2013) Improving decision making in multidisciplinary tumor boards: prospective longitudinal evaluation of a multicomponent intervention for 1,421 patients. J Am Coll Surg 217(3):412-420

14. Prades J, Remue E, van Hoof E, Borras JM (2015) Is it worth reorganising cancer services on the basis of multidisciplinary teams (MDTs)? A systematic review of the objectives and organisation of MDTs and their impact on patient outcomes. Health Policy 119:464-474

\section{Weiterführende Literatur}

15. Bundesgesundheitsministerium Nationaler Krebsplan Handlungsfeld 2: Weiterentwicklung der onkologischen Versorgungsstrukturen und der Qualitätssicherung. https:// www.bundesgesundheitsministerium.de/ themen/praevention/nationaler-krebsplan/ handlungsfelder/handlungsfeld-2.html. Zugegriffen:01.08.2020

16. Bundesgesundheitsministerium Nationaler Krebsplan Handlungsfeld 4: Stärkung der Patientenorientierung. https:// www.bundesgesundheitsministerium.de/ themen/praevention/nationaler-krebsplan/ handlungsfelder/ziele-des-nationalen-krebs plans.html.Zugegriffen:01.08.2020

17. El Saghir NS, Keating NL, Carlson RW et al (2014) Tumor boards: Optimizing the structure and improving efficiency of multidisciplinary management of patients with cancer worldwide. Am Soc Clin Oncol Educ Book34:e461-e466 
Hier steht eine Anzeige.

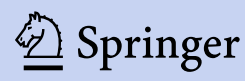

